\title{
Boar seminal plasma exosomes maintain sperm function by infiltrating into the sperm membrane
}

\author{
Jian Du ${ }^{1}$, Jian Shen ${ }^{1}$, Yuanxian Wang ${ }^{1}$, Chuanying Pan ${ }^{1}$, Weijun Pang ${ }^{1}$, Hua Diao ${ }^{2}$ \\ and Wuzi Dong ${ }^{1}$ \\ ${ }^{1}$ College of Animal Science and Technology, Northwest A\&F University, Yangling, Shaanxi, P. R. China \\ 2 NPFPC Key Laboratory of Contraceptives and Devices, Shanghai Institute of Planned Parenthood Research (SIPPR), \\ Institutes of Reproduction and Development, Fudan University, Shanghai, P. R. China
}

Correspondence to: Wuzi Dong, email: dongwuzi@126.com

Keywords: seminal plasma exosomes; boar sperm quality; capacitation; liquid storage; Pathology Section

Received: May 20, $2016 \quad$ Accepted: July 28, $2016 \quad$ Published: August 16, 2016

\section{ABSTRACT}

Seminal plasma ingredients are important for maintenance of sperm viability. This study focuses on the effect of boar seminal plasma exosomes on sperm function during long-term liquid storage. Boar seminal plasma exosomes had typical nanostructure morphology as measured by scanning electron microscopy (SEM) and molecular markers such as AWN, CD9 and CD63 by western blot analysis. The effect on sperm parameters of adding different ratio of boar seminal plasma exosomes to boar sperm preparations was analyzed. Compared to the diluent without exosomes, the diluent with four times or sixteen times exosomes compared to original semen had higher sperm motility, prolonged effective survival time, improved sperm plasma membrane integrity $(p<0.05)$, increased total antioxidant capacity (T-AOC) activity and decreased malondialdehyde (MDA) content. The diluent containing four times concentration of exosomes compared to original semen was determined to inhibit premature capacitation, but not to influence capacitation induced in vitro. Inhibition of premature capacitation is likely related to the concentration of exosomes which had been demonstrated to transfer proteins including AWN and PSP-1 into sperm. In addition, using fluorescence microscopy and scanning electron microscopy analysis, it was demonstrated that exosomes in diluent were directly binding to the membrane of sperm head which could improve sperm plasma membrane integrity.

\section{INTRODUCTION}

In order to become fully fertile after leaving testis, mammalian sperm must undergo morphological and functional changes during transit through epididymis and other accessory sex organs. Specifically, proteins and lipids on the sperm membrane are subtly modified by secretions of the male genital tract [1]. Upon ejaculation, spermatozoa are mixed with another set of surface re-modeling components derived from the accessory sex glands. Secretions from the male genital tract can influence the capacitation of sperm during their transit along the female genital tract [2]. Molecules on the surface of spermatozoa and in seminal plasma act in concert to stimulate or inhibit the onset and progression of the capacitation process $[3,4]$. It is thought that early capacitation-related events are accompanied with the loss, modification and redistribution of molecules on the sperm surface during sperm storage in vitro $[5,6]$. The decapacitation factors on sperm membranes and in seminal plasma are believed to stabilize the sperm membrane and keep sperm in a non-capacitated state, until these factors are removed during capacitation. It has also been observed that addition of ram or boar seminal plasma to sperm preparations improved viability and motility and reduced capacitation-like changes during sperm storage. Therefore, it is necessary to identify the components of boar seminal plasma that help to maintain sperm function during sperm storage in vitro.

Seminal plasma vesicles, which are characterized by high enrichment of cholesterol and sphingomyelin content, and a complex protein composition, have been isolated from the seminal plasma of human (namely prostasomes) [2, 7], rat [8], ram [9], and boar [10]. It 
Table 1: Effects of different concentrations of semen plasma exosomes on boar sperm motility (\%)

\begin{tabular}{|c|c|c|c|c|}
\hline \multirow{2}{*}{$\begin{array}{c}\text { Conservation } \\
\text { Time/day }\end{array}$} & \multicolumn{4}{|c|}{ Different concentration of semen plasma exosomes } \\
\cline { 2 - 5 } & Exo-0 & Exo-1 & Exo-4 & Exo-16 \\
\hline 0 & $83.69 \pm 2.46$ & $84.23 \pm 2.42$ & $84.012 \pm 3.95$ & $85.50 \pm 3.52$ \\
\hline 2 & $69.33 \pm 1.23 \mathrm{~b}$ & $70.17 \pm 1.89^{\mathrm{a}, \mathrm{b}}$ & $72.00 \pm 0.50^{\mathrm{a}}$ & $69.33 \pm 2.82^{\mathrm{b}}$ \\
\hline 4 & $58.33 \pm 1.25 \mathrm{~d}$ & $65.67 \pm 1.74^{\mathrm{c}}$ & $71.17 \pm 1.45^{\mathrm{a}}$ & $69.67 \pm 2.54^{\mathrm{b}}$ \\
\hline 6 & $50.30 \pm 1.89 \mathrm{c}$ & $60.00 \pm 1.21^{\mathrm{b}}$ & $64.12 \pm 1.53^{\mathrm{a}}$ & $64.17 \pm 1.76^{\mathrm{a}}$ \\
\hline 8 & $41.84 \pm 1.53 \mathrm{~d}$ & $56.33 \pm 1.23^{\mathrm{c}}$ & $62.00 \pm 0.96^{\mathrm{a}}$ & $60.17 \pm 0.87^{\mathrm{b}}$ \\
\hline 10 & $30.25 \pm 2.35 \mathrm{c}$ & $49.50 \pm 1.50^{\mathrm{b}}$ & $56.27 \pm 1.75^{\mathrm{a}}$ & $49.00 \pm 1.43^{\mathrm{b}}$ \\
\hline
\end{tabular}

Different alphabets superscripts within the same row demonstrate significant differences $(p<0.05)$, and the same alphabets superscripts demonstrate not significant $(p>0.05)$. Exo-0, Exo-1, Exo-4, Exo-16 represented the diluent with 0,14 and 16 times concentration of exosomes of original semen, respectively.

has been hypothesized that mammallian seminal plasma vesicles (perhaps including exosomes) can mediate decapacitation activity using several possible mechanism, such as inhibiting the capacitation-dependent cholesterol efflux, fluidity increase in sperm apical membranes, delivery of $\mathrm{Ca}^{2+}$ signaling molecule, and control of the acrosome reaction [11-13].

Seminal plasma exosomes are membrane vesicles ranging from 30 to $120-\mathrm{nm}$ diameter in size that are produced by organs in the male genital tracts including epididymis and prostate $[10,14,15]$. In boar, seminal plasma exosomes could inhibit the capacitation-dependent cholesterol efflux and mediate degradation of the 14$\mathrm{kD}$ phosphorylated polypeptide of capacitated sperm $[10,12]$. Several previous studies have demonstrated that seminal plasma exosomes may play an important role in maintaining the sperm function during in vitro preservation. However, the effect of boar seminal plasma exosomes as an additive to maintain semen quality during preservation at low temperature has not been reported. In the present study, the exosomes were isolated from Guanzhong-Black boar. Then the effect of exosomes on sperm motility, sperm membrane integrity, lipid peroxidation, and capacitation were investigated. Finally a possible modulatory role of exosomes on sperm function was firstly determined during long-term liquid storage of boar sperm at $17^{\circ} \mathrm{C}$ was discussed.

\section{RESULTS}

\section{Enrichment of boar seminal plasma exosomes}

To concentrate and identify boar seminal plasma exosomes, boar seminal plasma exosomes were isolated by ultracentrifugation using modified methods as previously described $[10,14]$. The exosome concentration of original seminal plasma (in Part I) were about $2 \times 10^{10}$ particles/mL as calculated by the NanoSight system. After concentration of these samples was calculated, different final exosome concentration in the diluent were adjusted to the exosomes concentration of original seminal plasma in the following experiments.

SEM demonstrated that some spherical membranous structures in the sample were 50-100 nm diameter membranous vesicles, within the size range of typical exosomes (Figure 1A, 1B). Furthermore, AWN, CD9 and $\mathrm{CD} 63$ in the membranous vesicles were proved by Western blot (Figure 1C).

\section{Boar seminal plasma exosomes prolonged effective motility time of sperm}

After the particle concentration was adjusted to the exosomes concentration of original seminal plasma, the effect of different concentrations of exosomes on boar sperm motility during liquid storage at $17^{\circ} \mathrm{C}$ was measured (Table 1). Sperm motility remained above $50 \%$ in the diluent (Exo-0) after 6 day of storage. For samples supplemented with Exo-1, Exo-4 and Exo-16 exosome additives, sperm motility was significantly higher than that of other groups after 8 days of storage ( $p$ $<0.05)$. The samples supplemented with Exo-4 exosomes maintained the highest motility of all groups after 10 days of storage $(p<0.05)$, but there was no difference between the groups with Exo-4 and Exo-16 addition $(p>0.05)$. These results indicated that Exo-4 and Exo-16 exosomes supplementation resulted in significantly prolonged sperm motility during storage.

\section{Boar seminal plasma exosomes improved plasma membrane integrity of spermatozoa}

The effects of different concentrations of exosomes on boar sperm plasma membrane integrity during liquid storage at $17^{\circ} \mathrm{C}$ is shown in Table 2 . After 2 days of storage, the plasma membrane integrity of the samples supplemented with Exo-1, Exo-4, and Exo-16 exosomes were significantly higher than that of other samples ( $p$ $<0.05)$. Furthermore, after 10-day of storage, samples supplemented with Exo-4, and Exo-16 exosomes 
Table 2: Effects of different concentrations of semen plasma exosomes on boar sperm plasma membrane integrity (\%)

\begin{tabular}{|c|c|c|c|c|}
\hline \multirow{2}{*}{$\begin{array}{c}\text { Conservation } \\
\text { Time/day }\end{array}$} & \multicolumn{3}{|c|}{ Different concentration of semen plasma exosomes } \\
\cline { 2 - 5 } & Exo-0 & Exo-1 & Exo-4 & Exo-16 \\
\hline 0 & $90.50 \pm 2.46^{\mathrm{c}}$ & $91.11 \pm 4.38$ & $92.43 \pm 2.95$ & $92.16 \pm 2.48$ \\
\hline 2 & $79.36 \pm 2.94^{\mathrm{c}}$ & $83.83 \pm 1.81^{\mathrm{b}}$ & $85.22 \pm 2.62^{\mathrm{a}, \mathrm{b}}$ & $87.43 \pm 3.16^{\mathrm{a}}$ \\
\hline 4 & $71.21 \pm 2.53^{\mathrm{c}}$ & $77.92 \pm 1.53^{\mathrm{b}}$ & $80.47 \pm 3.30^{\mathrm{a}}$ & $81.07 \pm 2.50^{\mathrm{a}}$ \\
\hline 6 & $61.84 \pm 3.35^{\mathrm{c}}$ & $69.89 \pm 3.95^{\mathrm{b}}$ & $75.15 \pm 3.46^{\mathrm{a}}$ & $74.37 \pm 1.63^{\mathrm{a}}$ \\
\hline 8 & $41.96 \pm 1.18^{\mathrm{c}}$ & $61.27 \pm 2.40^{\mathrm{b}}$ & $70.95 \pm 1.92^{\mathrm{a}}$ & $72.58 \pm 3.23^{\mathrm{a}}$ \\
\hline 10 & $35.20 \pm 2.88^{\mathrm{c}}$ & $55.30 \pm 3.39^{\mathrm{b}}$ & $66.10 \pm 3.94^{\mathrm{a}}$ & $67.96 \pm 3.74^{\mathrm{a}}$ \\
\hline
\end{tabular}

Different superscripts within the same row demonstrate significant differences $(p<0.05)$, and the same superscripts demonstrate not significant $(p>0.05)$. Exo-0, Exo-1, Exo-4, Exo-16 represented the extender with 0, 1, 4 and 16 times concentration of exosomes of original semen, respectively.

maintained more than $60 \%$ plasma membrane integrity, which was significantly higher than that of other groups $(p<0.05)$. However, there were no significant differences between the Exo-4 and Exo-16 samples $(p>0.05)$. The results indicated that supplementation with high concentration of exosomes (to at least the concentration of Exo-4) significantly improved the plasma membrane integrity of spermatozoa during long-term liquid storage at $17^{\circ} \mathrm{C}$.

\section{Determination of T-AOC activity and MDA content of sperm}

In order to analyze the antioxidant capacity of exosomes, T-AOC activity and MDA content of the samples supplemented with Exo-0, Exo-4, Exo-16 exosomes were determined during long-term liquid storage at $17^{\circ} \mathrm{C}$, respectively (Figure 2). Compared to samples without exosomes supplementation, decreased
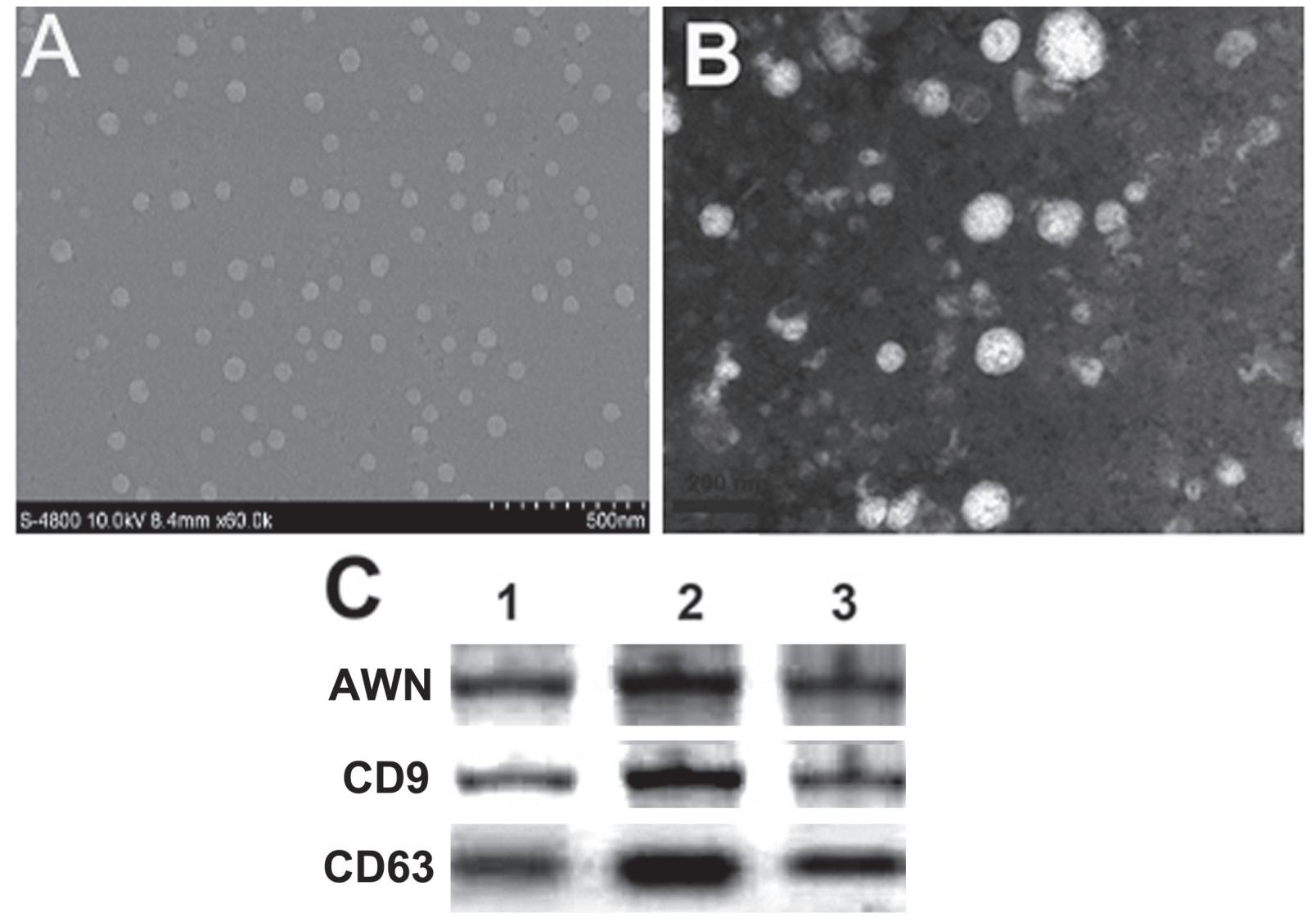

Figure 1: Identification of boar seminal plasma exosomes. A., B. Morphology of exosomes by scanning electron microscope. Morphology of negative stained exosomes (B); C. Molecular markers of exosomes (AWN, CD9 and CD63) were detected by Western blot. 1, 2, 3 lanes represented three repetitions. 
T-AOC activity and increased MDA content was observed in the groups supplemented with Exo-4 and Exo-16 ( $p$ $<0.05)$. T-AOC activity of the groups supplemented with Exo-4 was significantly lower after 6 days of incubation $(p<0.05)$ (Figure 2A) and MDA content was significantly higher after 8 day of incubation $(p<0.05)$ (Figure 2B) compared to samples with Exo-16 exosomes supplementation, respectively. The decrease of T-AOC activity and increase of MDA content accompanied by an increase of seminal plasma exosomes content indicated that Exo-4 might be the optimal concentration of exosomes for storage of sperm samples at $17^{\circ} \mathrm{C}$.

\section{Effect of boar seminal plasma exosomes on in vitro sperm premature capacitation}

In order to analyze the effects of exosomes on sperm capacitation in vitro, CTC staining was used to determine fluorescent patterns of sperm in the groups supplemented with Exo-0, Exo-4, and Exo-16 exosomes on day 4 of storage before and after sperm capacitation was induced using Tyrode's medium containing $3 \mathrm{mg} / \mathrm{mL}$ bovine serum albumin (BSA) at $37^{\circ} \mathrm{C}$ (Figure $3 \mathrm{~A}$ ). Before capacitation, the percentage of CTC staining fluorescent pattern A of the samples without exosomes was lower and that of pattern B was higher than other groups, but no difference was observed for pattern C. After capacitation, pattern A of sperm in the groups with Exo-16 exosomes was the highest and pattern $\mathrm{B}$ was the lowest compared with other groups, $(p<0.05)$. Notably, the rate of pattern A to pattern $\mathrm{B}, \mathrm{C}$ was not different between the groups with Exo-4 exosomes and the groups without exosomes, although pattern B of sperm in the groups with Exo-4 exosomes was the highest than others after induced capacitation. The same results appeared on day 8 of sperm storage in vitro (Figure 3B). These results indicated that exosomes could inhibit premature capacitation of sperm.

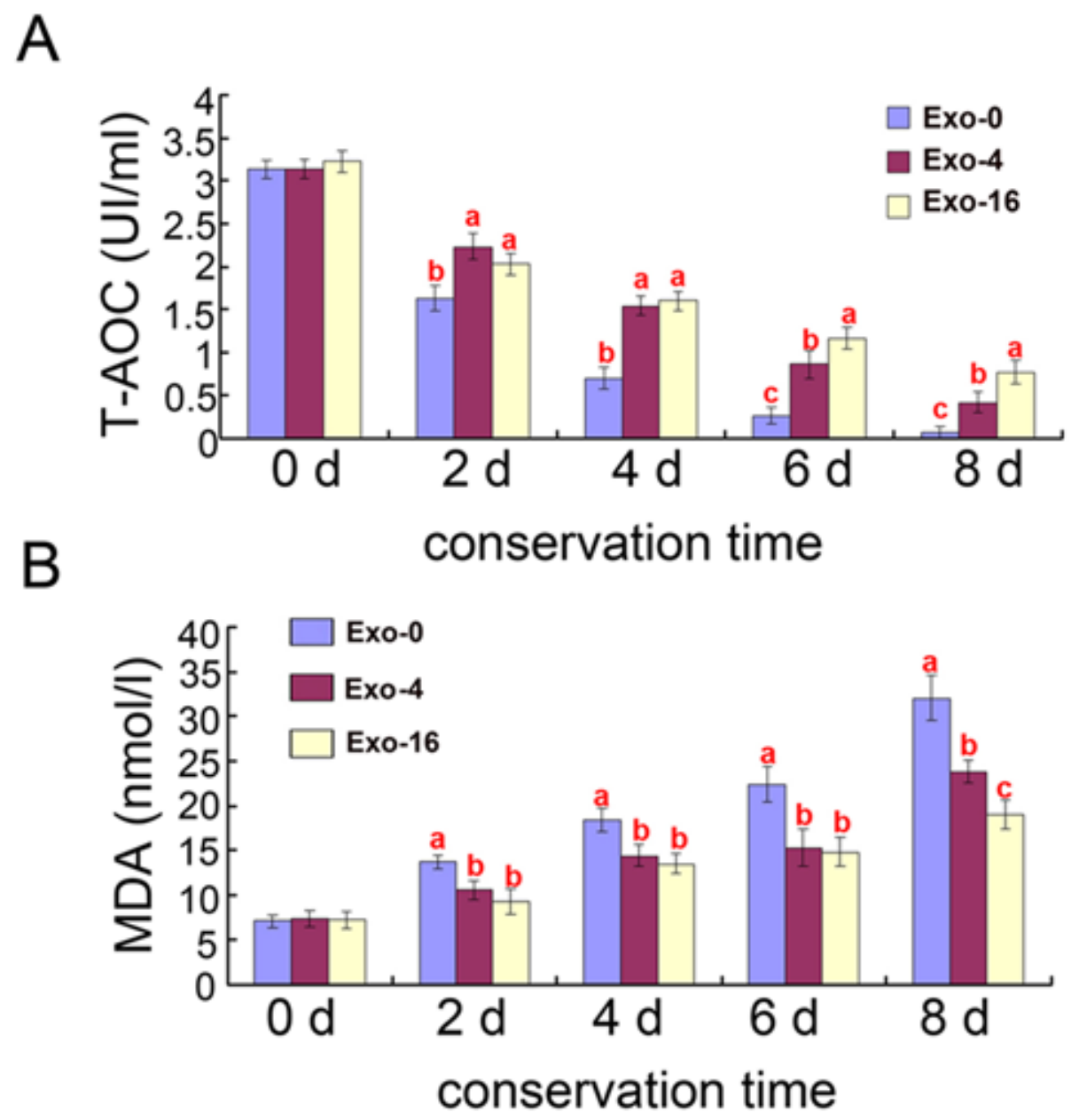

Figure 2: Effects of different concentrations of exosomes on T-AOC and MDA content in boar sperm during liquid storage at $17^{\circ} \mathbf{C}$. A. Effects of different concentrations of exosomes on T-AOC activity. B. Effects of different concentrations of exosomes on MDA content. Different superscripts within the same time demonstrate significant differences $(p<0.05)$, and the same superscripts demonstrate insignificant differences $(p>0.05)$. Exo-0, Exo-4, Exo-16 represented the diluent with 0, 4 and 16 times concentration of exosomes of original semen, respectively. 


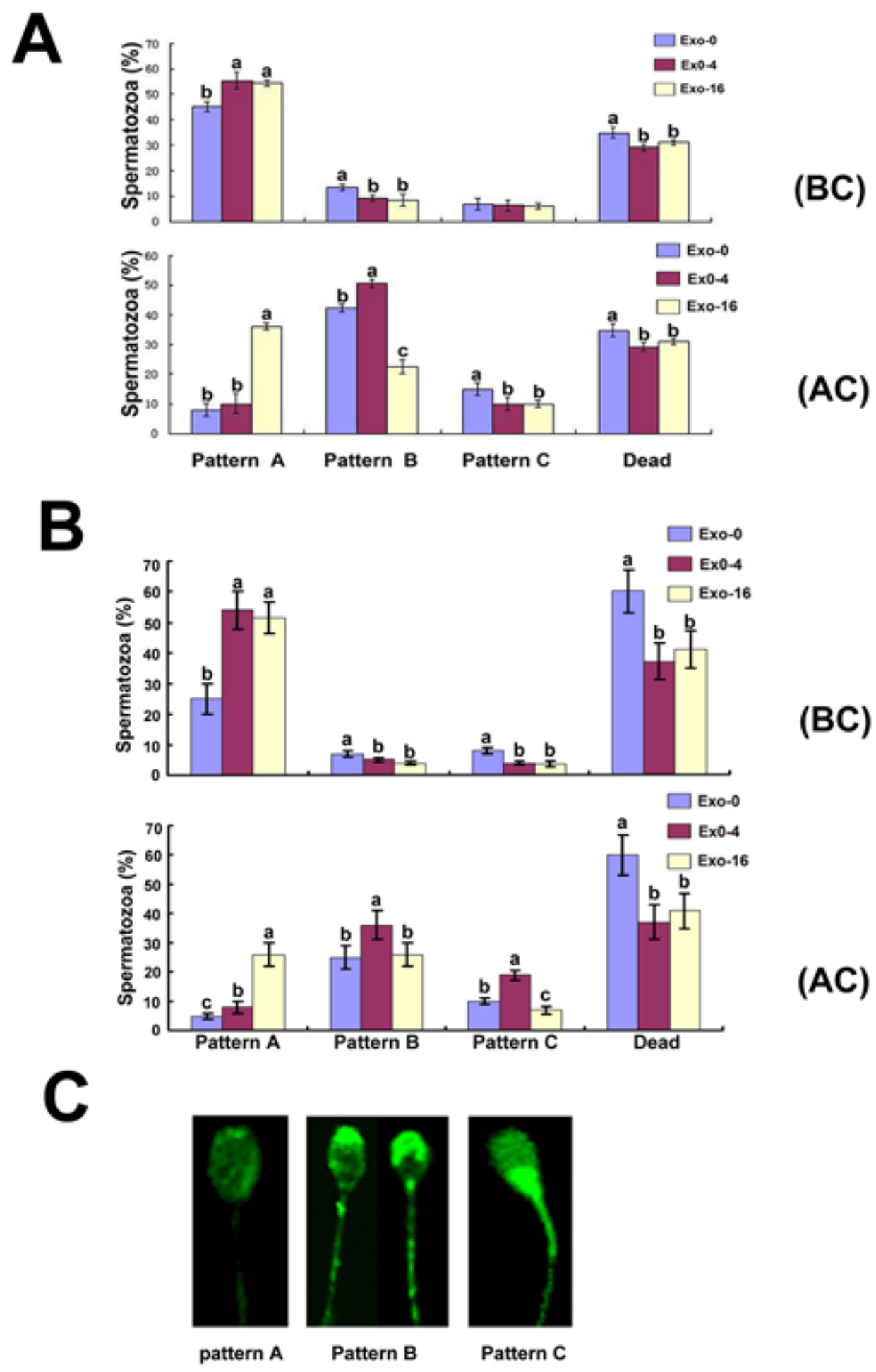

Figure 3: CTC staining patterns of sperm in the diluent with Exo-0, Exo-4 and Exo-16 exosomes. A, CTC staining patterns of sperm on the $4^{\text {th }}$ day in the diluent with Exo-0, Exo-4 and Exo-16 exosomes. (BC): before capacitation; (AC): at 3 hours after sperm capacitation was induced. B, CTC staining patterns of sperm on the $8^{\text {th }}$ day in the diluent with Exo-0, Exo-4 and Exo-16 exosomes. Capacitation fluid was Tyrode's medium with $3 \mathrm{mg} / \mathrm{mL}$ BSA (see Materials and Methods). (BC): before capacitation; (AC): at 3 hours after sperm capacitation was induced. C, illustration of spermatozoa different fluorescence patterns of spermatozoa samples. Pattern A. faint fluorescence uniformly distributed over the sperm head denoting non-capacitated spermatozoa. Pattern B. the acrosomal region of the sperm heads fluoresce brightly denoting capacitated spermatozoa. Pattern C. the acrosomal region of the sperm head is non-fluorescent while the post-acrosomal region of the sperm head appeared strong fluorescence and this indicated a capacitated, acrosome-reacted spermatozoa. Dead represented the percentage of spermatozoa with no movement of the flagellum. Exo-0, Exo-4, Exo-16 represented the diluent with 0 , 4 and 16 times concentration of exosomes of original semen, respectively. Sperm head length: $7.0 \mu \mathrm{m}$. 
Analysis of AWN and PSP-1 on sperm during in vitro storage

AWN and PSP-1 which are highly abundant proteins in boar seminal plasma have been observed to load in exosomes $[10,16,17]$. To analyze changes of proteins on the sperm membrane, immunofluorescence was used to detect the location of AWN and PSP-1 on sperm on day 0 , day 4 , and day 8 of sperm storage in the samples with Exo-0, Exo-4, or Exo-16 supplementation (Figure 4 \& 5). AWN was primarily localized on the acrosome membrane (Figure 4A, 4B, 4C) as the same as previous reports $[16,17]$ have reported and PSP-1 proteins appeared simultaneously on acrosome membranes and in the apical area of the middle piece of the sperm tail (Figure 5A, 5B,
5C), confirming results previously reported by Caballero Ignacio et al [18]. Loss of AWN and PSP-1 from the acrosome membrane was slower in the sample with Exo4 exosomes than in the basic diluent without exosomes (Figure 4 and Figure 5). Notably, by day 8, the majority of spermatozoa lose AWN (Figure 4G) and PSP-1 (Figure $5 \mathrm{G})$ from acrosome membrane in the diluent, but partial recovery of AWN and PSP-1 on the sperm membrane was observed when exosomes was added into the diluent for 2 days (Figure 4G insert and Figure 5G insert). Conversely, PSP-1 protein remained only on the apical area of the middle piece of sperm tail (Figure 5). However, remnants of AWN and PSP-1 still appeared on acrosome membranes on day 8 when sperm was incubated in the basic diluent with Exo-4 or Exo-16 (Figure 4 and Figure 5).
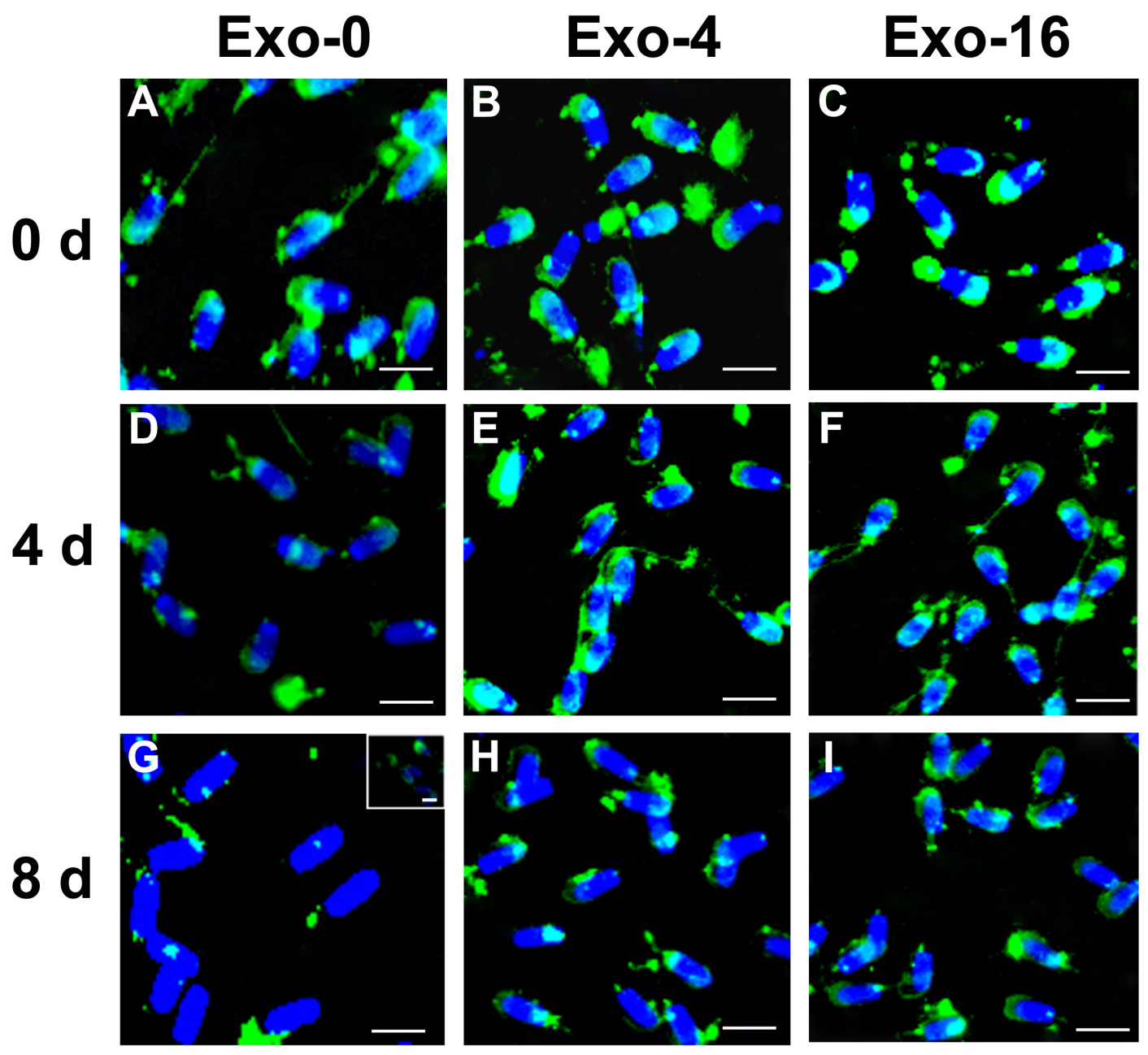

Figure 4: Immunofluorescence detection of AWN on sperm incubated in the diluent without exosomes (Exo-0) and with different concentrations of exosomes (Exo-4 and Exo-16) on day 0, and after 4 days and 8 days at $17^{\circ} \mathrm{C}$. In the diluent without exosomes (Exo-0), AWN was present on the acrosome membrane of all sperm on day 0 A. Partial AWN protein existed on acrosome membrane of sperm $\mathbf{D}$. All sperm had lost AWN on acrosome membrane by day $8 \mathbf{G}$. In the diluent with different concentrations of exosomes (Exo-4 and Exo-16), respectively, AWN located on the acrosome membrane of all sperm on day 0 B. and $\mathbf{C}$. and day $4 \mathbf{E}$. and F. AWN dispersed in front area of sperm heads on day $8 \mathbf{H}$. and $\mathbf{I}$. Arrowheads represented AWN location on acrosome membrane. Exo-0, Exo-4 and Exo-16 represented the diluent without exosomes, the diluent with four times exosomes concentration, and sixteen times exosomes concentration of original semen, respectively. Sperm head length: $7.0 \mu \mathrm{m}$. 


\section{Analysis of binding of boar seminal plasma exosomes with spermatozoa}

To analyze binding or fusing of exosomes with spermatozoa, the sperm samples were observed using fluorescence microscopy after treatment by $0.125 \%$ trypsin. Spermatozoa with DiI-exo display red fluorescence under fluorescence microscope (Figure 6). Few sperm were observed with fluorescent staining in the diluent with DiI-exo (Exo-1) exosomes (data not shown). For sperm samples incubated in the diluent containing DiIexo (Exo-4 or Exo-16) at $17^{\circ} \mathrm{C}$, about $50 \%$ of the sperm showed fluorescence after one day of storage (Figure 6A, 6B). Furthermore, all sperm displayed fluorescence on day 4 of storage (Figure 6C,6D). These results showing that binding of exosomes with spermatozoa is positively correlated to the concentration of exosomes and incubation time indicate that exosomes may directly bind to the sperm membrane.

In order to further analyze the effect of exosomes on sperm membranes, SEM was used to observe the ultrastructure of sperm on day 4 of storage under various different conditions (Figure 7). The results of SEM showed that the number of nano-granules (exosomes) on the membrane surface of sperm in the diluent with Exo-1 were fewer than those in the diluent with Exo-4 or Exo16 exosomes. The exosomes were found to be distributed densely on the front of sperm heads and the number of exosomes on the surface of sperm membranes was not significantly different in the diluent with Exo-4 or Exo-16.
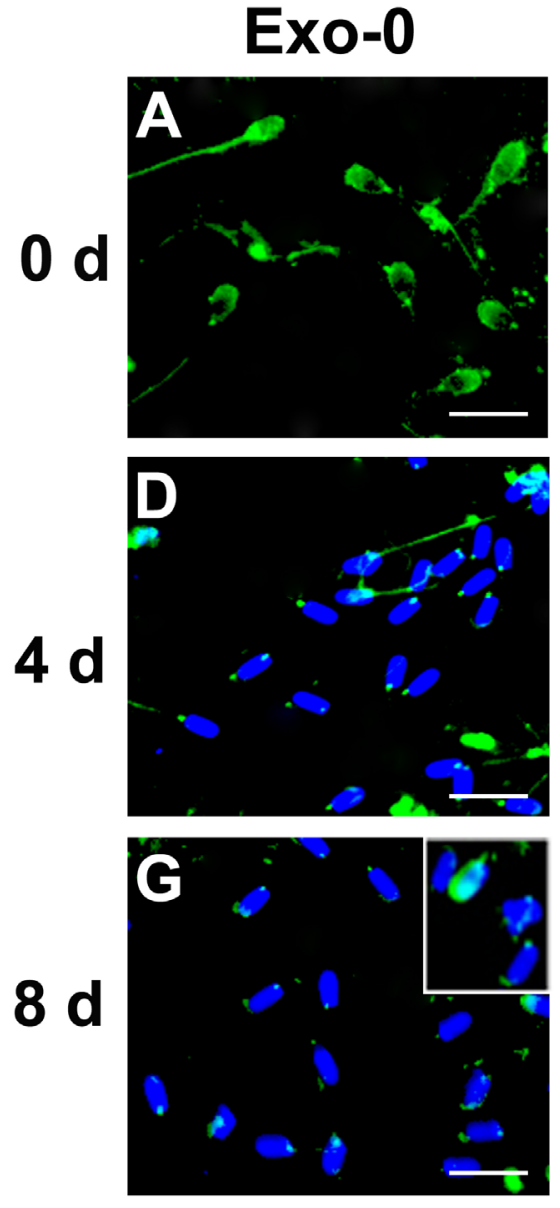

Exo-4
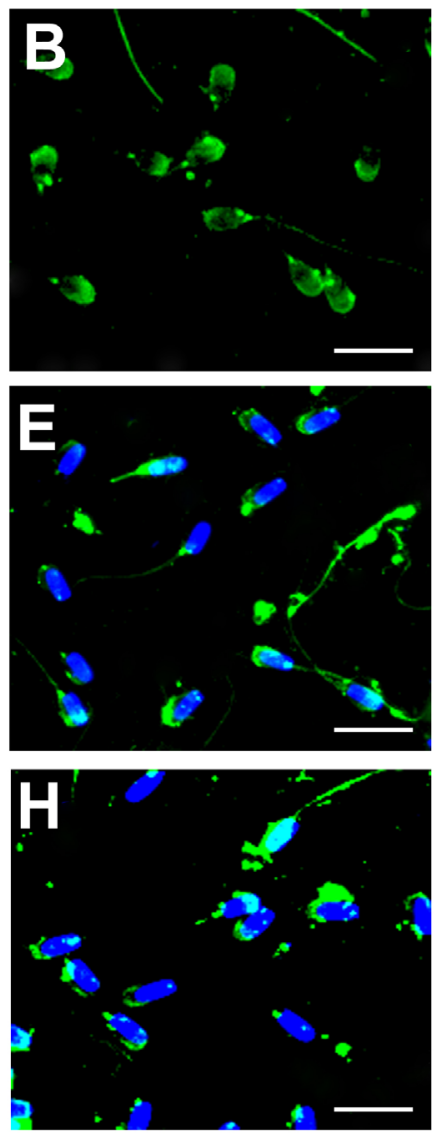

Exo-16
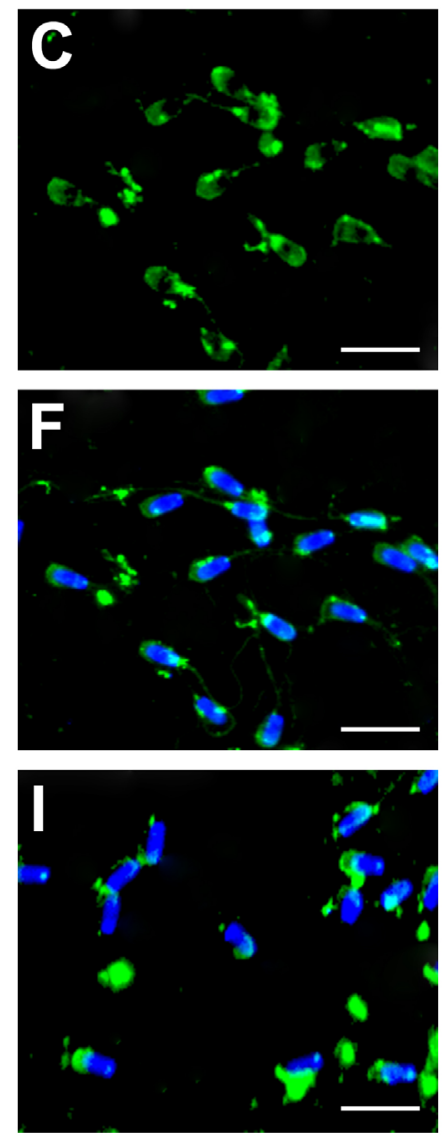

Figure 5: Immunofluorescence detection of PSP-I on sperm incubated in the diluent without exosomes (Exo-0) and with different concentrations of exosomes (Exo-4 and Exo-16) on day 0 , and after 4 days and 8 days at $17^{\circ} \mathrm{C}$. In the diluent without exosomes, PSP-1 located on acrosome membrane and apical area of sperm tail on day 0 A. Partial PSP-1 protein existed on the acrosome membrane and principal area of sperm tail on day $4 \mathbf{D}$. All sperm had lost PSP-1 on acrosome membrane and only remain PSP-1 remained in the apical area of the middle piece of sperm tail on day 8 G.. In the diluent with different concentrations of exosomes (Exo-4 or Exo-16), respectively, PSP-1 existed on the acrosome membrane and principal areas of the sperm tail on day 0 B., C. and day 4 E., F. and sperm lost part of the PSP-1 on the acrosome membrane on day 8 H., I. Arrowheads and asterisk represent PSP-1 locations on acrosome membranes and on the apical area of the middle piece of sperm tails, respectively. Exo-0, Exo-4 and Exo-16 represented the diluent without exosomes, four times exosomes concentration and sixteen times exosomes concentration of original semen, respectively. Sperm head length: $7.0 \mu \mathrm{m}$. 


\section{DISCUSSION}

Mammalian seminal plasma originates from multiple cellular sources in the male genital tract including testis, epididymis, vas deferens, vesicular glands, prostate gland and other accessory sexual glands. Seminal plasma contains a high concentration of subcellular lipid-bound microparticles (extracellular vesicles) which have been identified to encompass a complex composition of lipids, mRNAs, microRNAs and proteins [12, 19]. Exosomelike vesicles have also been isolated from the seminal plasma of rat [20], rabbit [21], ram [22], bull [23],

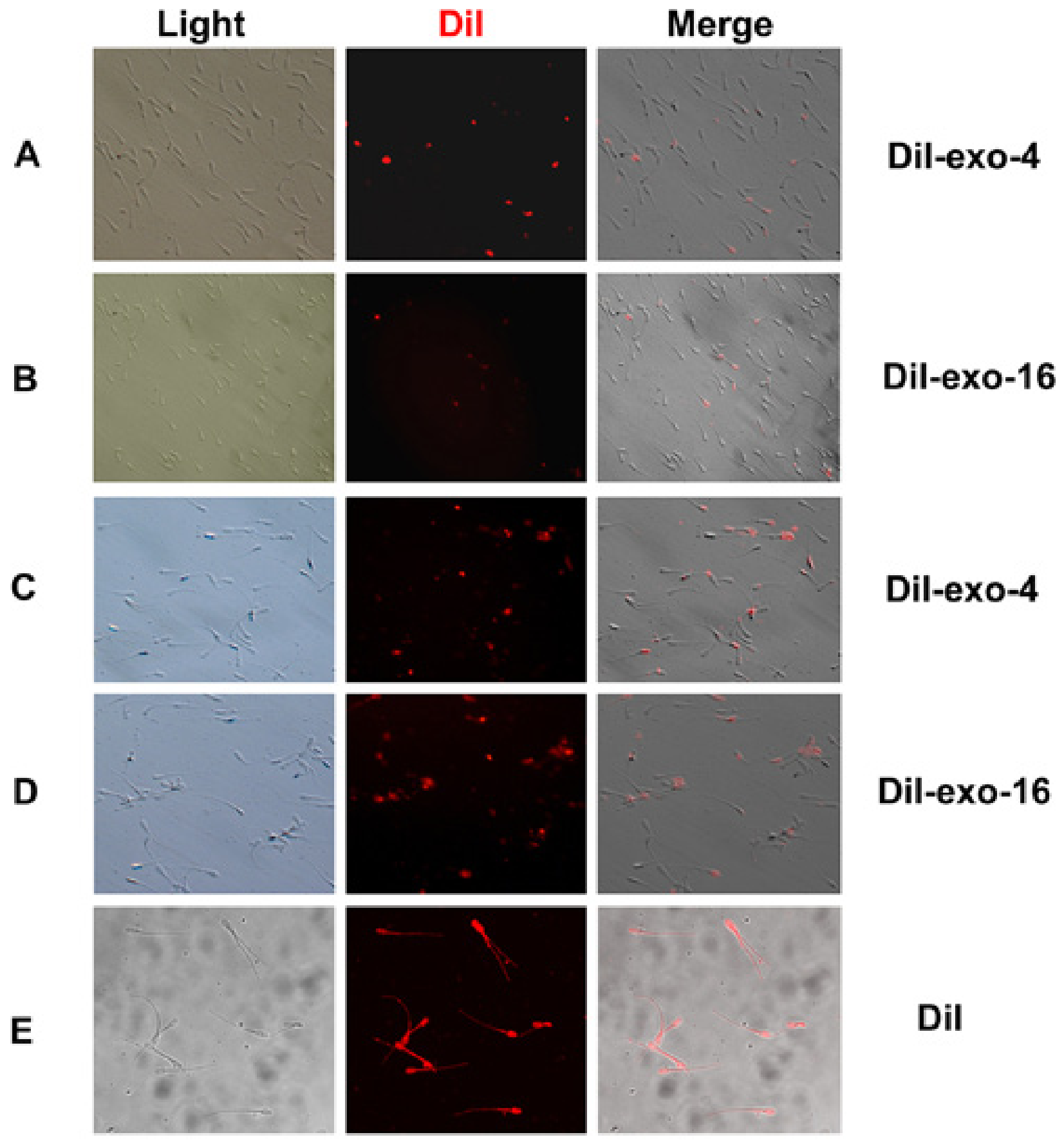

Figure 6: Red fluorescence sperm was detected during incubation with different concentration of DiI-exo (DiI-exo-4 and DiI-exo-16) at $1^{\circ} \mathrm{C}$. The sperm samples were treated by $0.125 \%$ trypsin and then washed 3 times using the diluent. Finally, the 2 $\mu \mathrm{L}$ of sperm samples were dropped on cover slides and observed using fluorescence microscopy. A. Sperm was incubated one day in the diluent with DiI-exo-4; B. Sperm was incubated one day in the diluent with DiI-exo-16; C. Sperm was incubated 4 days in the diluent with DiI-exo-4; D. Sperm was incubated 4 days in the diluent with DiI-exo-16. E. Sperm incubated 3 hours in the diluent with $0.005 \mathrm{mM}$ DiI dye displayed red fluorescence. DiI-exo-4, DiI-exo-16 represented the diluent with four and sixteen times concentration of exosomes of original semen, respectively. And the exosomes were labeled DiI dye. Scale: sperm head length $7.0 \mu \mathrm{m}$. 
stallion [24], and boar [10]. Mammalian seminal plasma exosomes share common characteristics, though functions of the accessory sex glands vary among mammalian species and this may well affect the nature and relative proportions of the extracellular vesicles. In the current study, exosomes isolated from boar seminal plasma were lipid-bound microparticles ranging in size from 30-100 $\mathrm{nM}$ in diameter, similar to other reports (Figure 1A, 1B). Molecular markers such as AWN, CD63 and CD9 were also found to be present in the exosomes as demonstrated by Western blot (Figure 1C).

In all studies so far, exosomes have been shown to be cell-derived vesicles that are present in many (perhaps all) biological fluids, including urine, blood, semen, ascites, and cerebrospinal fluid [25]. It is increasingly clear that most vesicles have specialized functions and play a key role in intercellular signaling and the immune system [26]. Mammallian seminal plasma exosomes could have extensive roles in fertilization biology. Seminal plasma exosomes inhibited the capacitation-dependent cholesterol efflux and fluidity increase in apical membranes, and 14$\mathrm{kD}$ polypeptide phosphorylation [10]. Human prostasomes including exosomes have been shown to play important roles in increasing sperm motility, affecting the tyrosine phosphorylation of sperm proteins and delaying acrosomal reaction, and impacting sperm-oocyte interaction by delivering related proteins to sperm $[19,27,28]$. It has been suggested that seminal plasma exosomes may play an important role in maintaining the sperm function during sperm in vitro preservation of sperm samples.

Liquid storage of boar semen is widely used in commercial swine artificial insemination. Sperm motility is one of the key factors in the conservation of sperm. Different additives including some single exogenous chemical reagents and endogenous components of seminal plasma added into sperm diluent could promote semen quality during cryopreservation. Interestingly, the addition of boar seminal plasma with good sperm freezability to the freezing extender can improve boar sperm cryosurvival [29]. However, the supplementation of the freezing extender with endogenous PSP-I/PSP-II did not affect post-thaw sperm survival [30]. Furthermore, human prostasomes have been shown to play an important role in increasing sperm motility [31]. In our study, boar semen plasma exosomes did improve the sperm motility during storage in $17^{\circ} \mathrm{C}$ (Table 1). With the optimal concentration of exosomes (Exo-4) in the diluent, the effective preservation time was longer than 10 days (motile sperm
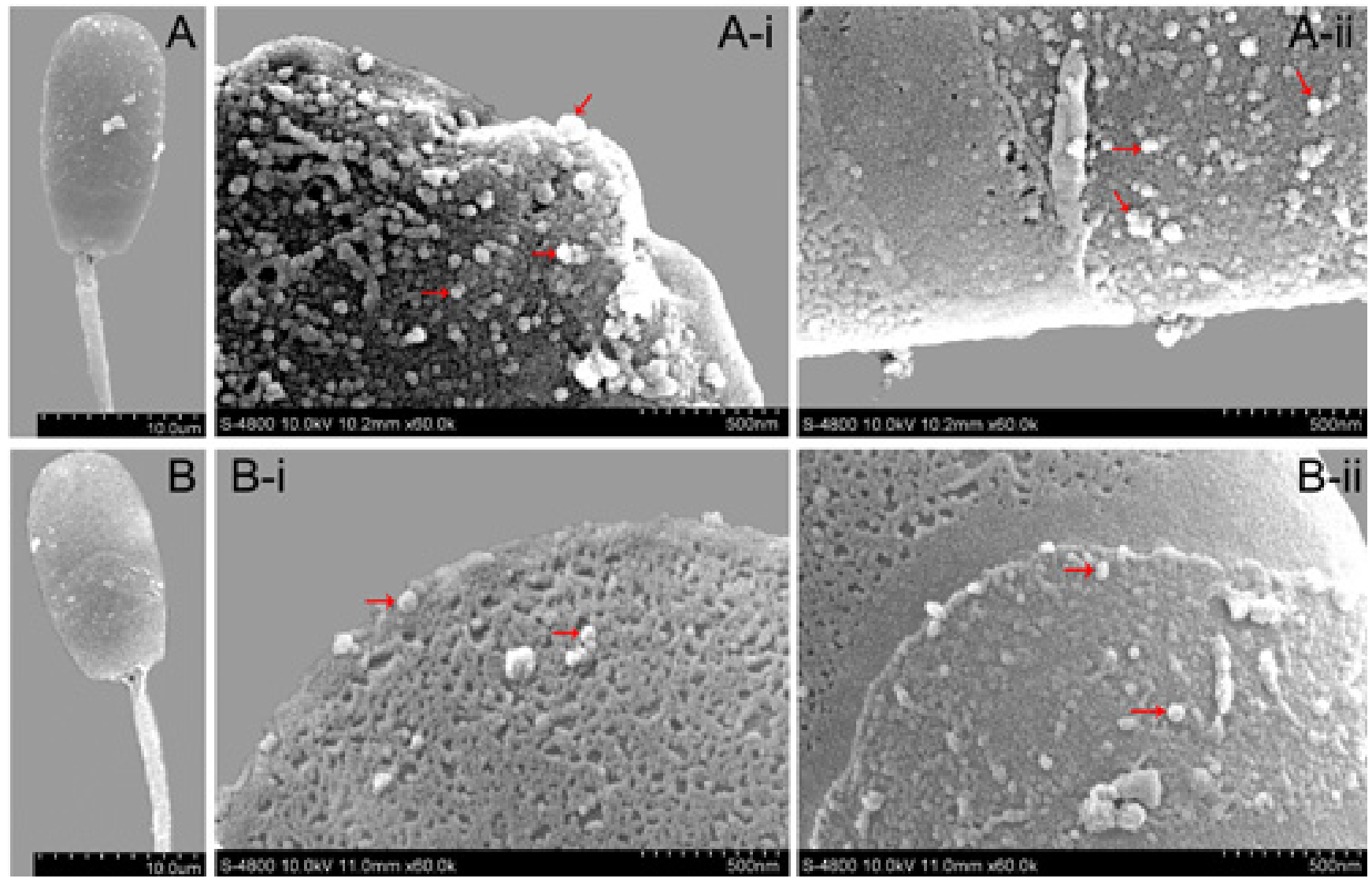

Figure 7: The ultrastructure of sperm at $4 \mathbf{d}$ in different conditions by scanning electron microscopy (SEM). A. A-i, A-ii: Ultrastructure of membrane surface of sperm head in the diluent with Exo-1. B. B-i, B-ii: Ultrastructure of membrane surface of sperm head in the diluent with exosomes (Exo-4 or Exo-16). Arrowheads indicated the nano-granules (exosomes). 
$>50 \%$ ), and sperm motility remained at $65 \%$ after 8 days of storage.

The sperm plasma membrane is an outer cell structure that acts as a physiological barrier to maintain sperm architecture. Proteins and lipids in seminal plasma contribute to maintenance of sperm membrane integrity maintenance which is required for normal sperm activities and directly reflects the degree of sperm injury. Sperm membrane integrity is also highly correlated with sperm motility and survival during sperm storage [32, 33]. In our study, the plasma membrane integrity of sperm was significantly higher in diluents supplemented with exosomes than that of control samples after 2 days of storage at $17^{\circ} \mathrm{C}(p<0.05)$, but plasma membrane integrity of the groups supplemented with Exo-4 and Exo-16 exosomes was not significantly different until 10 days' preservation $(p>0.05)$ (Table 2$)$. These results indicate that certain concentrations of exosomes significantly improved the plasma membrane integrity of spermatozoa during long-term liquid storage.

Oxidative stress can induce peroxidation of the sperm plasma membrane to affecting the physiology of sperm [34]. The broken balance of ROS generation and scavenging activity is disrupted leading to excessive ROS generation during boar sperm storage at room temperature [35]. The decrease of sperm motility could be explained by peroxidative damage of excessive ROS. MDA, a byproduct of lipid peroxidation, has been used to monitor the degree of peroxidative damage sustained by spermatozoa and T-AOC activity is another indicator to detect sperm membrane antioxidant capacity $[36,37]$. The current study demonstrating increased T-AOC activity and decreased MDA content in the diluent supplemented with exosomes indicated exosomes can elevate the antioxidant capacity of sperm samples during storage (Figure 2). Exosomes concentration was also positively correlated with sperm motility (Table 1) and plasma membrane integrity of boar sperm during liquid storage (Table 2). These results indicate that exosomes could enhance antioxidation properties of sperm by maintaining the plasma membrane integrity of sperm.

Generally, an increase of $\mathrm{Ca}^{2+}$ influx to sperm is an indicator of characterized capacitation status [3840]. CTC is used to detect $\mathrm{Ca}^{2+}$ accumulation in certain organelle membranes and sperm is labeled with CTC at those parts of the surface membranes where $\mathrm{Ca}^{2+}$ is present above a certain concentration to allow CTC stain [41-43]. Inhibition of premature capacitation is essential to sperm

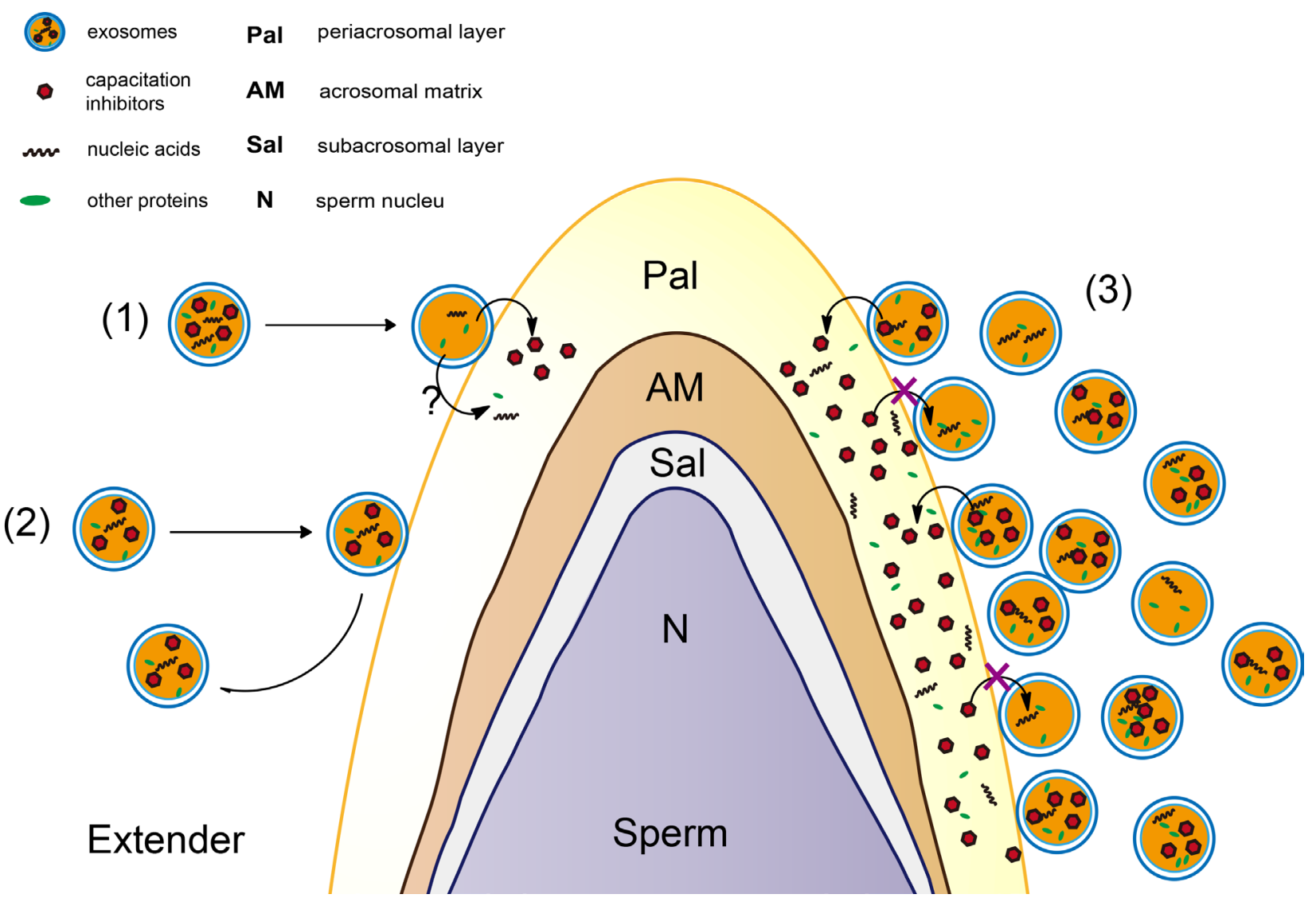

Figure 8: A speculative model of exosomes for protection of sperm function. (1) It was a dynamic process that exosomes was released from and band with sperm membrane. (2) The elements in exosomes could be accumulated in sperm through exosomes transferring into membrane of sperm. (3) Excessive exosomes in diluent led to transfer more exosomes ingredients such as AWN and PSP-1 proteins (as capacitation inhibitors) into sperm membrane in vitro. 
survival during long-term in vitro sperm preservation. Compared to the sperm samples without exosomes, inhibition of sperm premature capacitation was enhanced with an increased concentration of exosomes in the diluent at $17^{\circ} \mathrm{C}$ (Figure $3 \mathrm{~A}$ ). Interestingly, compared to the sperm that was incubated in the diluent without exosomes for 4 days at $17^{\circ} \mathrm{C}$, the capacitation of sperm with the diluent containing Exo-4 was not significantly different $(p>$ $0.05)$, but the capacitation of sperm in the diluent with Exo-16 was significantly decreased $(p<0.05)$ when sperm capacitation was induced by $3 \mathrm{mg} / \mathrm{ml} \mathrm{BSA}$ (Figure 3B).

Some components in seminal plasma modulate decapacitation to influence fertilization ability of spermatozoa, and are delivered via small vesicles in seminal plasma. Consecutive complement of AWN and PSP-1 to sperm could inhibit premature capacitation [44]. In our results, remnants of AWN and PSP-1 still appeared in acrosome membranes on day 8 of storage when sperm was incubated in the diluent with Exo-4, and the similar results were observed in the diluent with Exo-16 (Figure 4 and Figure 5). Changes in AWN and PSP-1 on the sperm membrane indicated that exosomes in the diluent could help to maintain sperm function perhaps via transmission of decapacitation factors (such as AWN and PSP-1) to sperm during long-term liquid storage (Figure 4 and Figure 5).

It is well known that cholesterol and phospholipids are essential components of mammalian cell membranes including exosomes. Consequently, cellular membrane integrity can be maintained by balancing the physiological cholesterol/phospholipid ratio [45]. Numerous studies indicate that the interaction between cholesterol and phospholipid could prevent the premature capacitation through spermatozoa membrane stabilization [46]. Vesicles (including exosomes) could inhibit progression to late capacitation events and avoid premature acrosome reaction by binding or fusion to sperm membrane early during capacitation [11]. In our study, we observed that exosomes permeate into sperm (Figure 6 and Figure 7). An interesting observation was that almost all of the sperm absorbed the DiI-exo in diluent with Exo-4 or Exo-16 exosomes after 4 days of liquid storage sperm at $17^{\circ} \mathrm{C}$ (Figure $\left.6 \mathrm{C}, \mathrm{D}\right)$. Furthermore, SEM revealed a large number of nano-scale granules on the sperm head membrane in the diluent with exosomes (Exo-4 or Exo16) (Figure 7A, A-i, A-ii). In contrast, few nano-scale granules were observed on the sperm head membranes in the diluent with Exo-1 (Figure 7B, B-i, B-ii). One possible explanation is that exosomes are in a state of equilibrium inside and outside the membrane of sperm in the condition of certain concentrations of exosomes (such as Exo-4) and the balance is disturbed when excessive exosomes are added, leading to exosomes transferring more proteins into sperm including factors that inhibit capacitation (Figure 8).

\section{CONCLUSIONS}

In this study, we report that boar seminal plasma exosomes were essential in maintaining sperm motility, sperm membrane integrity, antioxidant capacities and inhibition of premature capacitation. It was first determined that boar seminal plasma exosomes could protect sperm function through binding to the sperm membrane and transferring proteins (such as AWN and PSP-1) to sperm. Our model for exosome-mediated protection of sperm includes three speculative possibilities (Figure 8): (1) It could be a dynamic process in which exosomes are released from and bound to the sperm membrane. (2) Endogenous proteins in exosomes could accumulate in sperm through exosomal transfer. (3) Excessive exosomes in diluent could lead to transfer of more exosomal proteins such as AWN and PSP-1 into the boar sperm membrane in vitro. Due to the heterogeneity of exosomes derived from different organs in the genital tract, it will be necessary in future research to investigate the effect on sperm preservation of seminal plasma exosomes derived from different organs of the genital tract. It will also be interesting to determine which components in exosomes (including special proteins, lipids and miRNAs) are necessary to preserve the viability and function of porcine sperm.

\section{MATERIALS AND METHODS}

\section{Semen collection}

Semen samples from eight sexually mature and healthy Guanzhong-Black boars (2.5-3 years old) were used in this study. Individual ejaculates were collected into pre-warmed tubes twice a week by the gloved hand technique before transportation to the laboratory. Each sample was assessed for sperm concentration using a calibrated spectrophotometer and the percentage of motility was evaluated at $37^{\circ} \mathrm{C}$ using a phase-contrast microscope. Only the semen samples with more than $10^{7}$ spermatozoa $/ \mathrm{ml}$ and $80 \%$ motility were selected and used for this study. The sperm-rich fractions were pooled and transferred to the laboratory within half an hour. A total of 40 ejaculates ( 5 ejaculate samples per boar) from eight boars were used in this study.

Semen samples were processed according to a standard cryopreservation protocol with some modifications [47]. Briefly, the semen samples were centrifuged ( $800 \times g, 10 \mathrm{~min}$ at room temperature) to separate sperm and supernatant. The supernatant was retained for purification of exosomes. The sperm pellets were re-suspended to a final concentration of $5 \times 10^{7}$ spermatozoa $/ \mathrm{mL}$ in the diluent supplemented with exosomes of different final concentrations at $17^{\circ} \mathrm{C}$. The 
diluent was composed of $27.5 \mathrm{~g}$ of glucose, $2.35 \mathrm{~g}$ of EDTA- $\mathrm{Na}_{2}, 6.9 \mathrm{~g}$ of sodium citrate, $2.9 \mathrm{~g}$ of citric acid, 1.0 $\mathrm{g}$ of $\mathrm{NaHCO}_{3}, 6.65 \mathrm{~g}$ of Tris for per $1000 \mathrm{ml}$ of deionized water $(\mathrm{pH}=7.2)[48]$.

\section{Isolation and analysis of exosomes from boar seminal plasma}

\section{Isolation of exosomes from boar seminal plasma}

Boar seminal plasma exosomes were isolated by ultracentrifugation according to a previously described method with modifications method of previous report [10]. In each experiment, $300 \mathrm{ml}$ of the semen supernatant was centrifuged at $16,000 \times g$ for $1 \mathrm{~h}$ at $4^{\circ} \mathrm{C}$ to remove cell debris. The supernatant was filtered using $0.2 \mu \mathrm{m}$ filters (the semen was named Part I), and centrifuged at 120,000 $\times g$ for 1 hour at $4^{\circ} \mathrm{C}$. The pellets were then washed twice with buffer (30 mM TRIS, $130 \mathrm{mM} \mathrm{NaCl}, \mathrm{pH} 7.6$ ) followed by centrifugation at $120,000 \times g$ for $1 \mathrm{~h}$ at $4^{\circ} \mathrm{C}$. Finally, the pellet was resuspended in $3 \mathrm{ml}$ of the diluent.

\section{DiI labeling of exosomes}

Exosomes were dissolved in PBS and $0.01 \mathrm{mM}$ DiI dye in alcohol was added, and then the solution was incubated for $30 \mathrm{~min}$ at $37^{\circ} \mathrm{C}$. The solution was centrifuged at $12,000 \times \mathrm{g}$ for $30 \mathrm{~min}$ and the supernatant was filtered using $0.2 \mu \mathrm{m}$ filters. Excess DiI dye was removed by centrifugation at $120,000 \times g$ for 1 hour at $4^{\circ} \mathrm{C}$ and the pellet (the DiI-labeled exosomes abbreviated as DiI-exo) was resuspended with the diluent.

\section{Observation of exosomes}

Samples (Part I, the filtered seminal supernatant and DiI-exo) detection and counting were performed on the NanoSight NS300 following manufacturer's protocols (Malvern Instruments, Malvern, UK). Particle concentration (particles $/ \mathrm{mL}$ ) was calculated by the NanoSight system. Samples of exosomes were observed by electron microscopy using previously described methods. The concentration of exosomes in Part I was the concentration of exosomes in the original seminal plasma.

\section{Semen processing}

Sperm pellets were diluted in the diluent (to $\sim 10^{7}$ spermatozoa $/ \mathrm{ml}$ concentration) for the control group (no exosomes, Exo-0), and other groups including Exo-1 (the diluent with $1 \times$ exosomes concentration of the original seminal plasma), Exo-4 (the diluent with $4 \times$ exosomes concentration of original semen), and Exo-16 (the diluent with $16 \times$ exosomes concentration of original semen), respectively. Finally, semen samples ( $\sim 10^{7}$ spermatozoa/ $\mathrm{ml}$ concentrations) were all stored in the incubator at $17^{\circ} \mathrm{C} \pm 0.1^{\circ} \mathrm{C}$ in an atmosphere $5 \% \mathrm{CO}_{2}$ and saturated humidity.
In order to observe exosomes interacting with spermatozoa, the experiments were conducted using the following procedure. Briefly, varying concentrations of DiI-labeled exosomes were added into the diluent. At different time points during sperm incubation at $17^{\circ} \mathrm{C}$, the sperm were collected by centrifugation at $800 \times g, 10 \mathrm{~min}$ at $17^{\circ} \mathrm{C}$. Sperm were washed 3 times with the pre-cooled diluent. Finally, sperm in the diluent dropped on a clean slide were observed under fluorescent microscope. At least three separate experiments (consisting of three replicates) were conducted for each concentration of exosomes.

\section{Analysis of sperm motility}

The percentage of motile sperm was assessed visually every 2 days using a phase-contrast microscope (Nikon, Tokyo, Japan), equipped with a warm stage. After 5 min of incubation at $37^{\circ} \mathrm{C}, 5 \mu \mathrm{l}$ of every semen sample was placed on a glass slide and covered by a glass coverslip. Sperm motility estimations were performed in three different microscopic fields for each semen sample. Sperm motility was evaluated by visual estimation. Ten $\mu \mathrm{L}$ of sperm suspension was delivered onto a pre-warmed clean glass slide, and covered with a clean coverslip. The slides were examined under an optical microscope with a bright field (Nikon 80i; Tokyo, Japan) at 200× total magnification. The percentages of sperm showing progressive movement were estimated and noted after viewing five different fields. Three separate aliquots (replicates) were assessed from each semen sample. The mean of the three successive estimations was recorded as the final sperm motility $(\%)$.

\section{Analysis of sperm membrane integrity}

Sperm membrane integrity was evaluated using fluorescent probes [49]. Briefly, The SYBR-14 was prepared in anhydrous dimethyl sulfoxide (DMSO) at a concentration of $0.1 \mathrm{mg} / \mathrm{ml}$. Propidium iodide (PI) was dissolved in PBS at $2 \mathrm{mg} / \mathrm{ml}$. Aliquots $(500 \mu \mathrm{L})$ of sperm were stained at $37^{\circ} \mathrm{C}$ with $0.27 \mu \mathrm{L}$ of SYBR-14 and $2 \mu \mathrm{L}$ of PI solution. The samples were incubated for $15 \mathrm{~min}$ at $37^{\circ} \mathrm{C}$ before examination. Sperm staining was monitored and photographed at $400 \times$ magnification by an epifluoresence microscope (Nikon 80i; Tokyo, Japan) using filters set to $535 \mathrm{~nm}$ excitation and $617 \mathrm{~nm}$ emission for PI red fluorescence and $488 \mathrm{~nm}$ excitation and $516 \mathrm{~nm}$ emission for SYBR-14 green fluorescence. For the same field, photographs were also taken with a phase-contrast microscope. At least 200 spermatozoa per slide were counted. The mean of three successive estimations was recorded as the final sperm membrane integrity score (\%). 


\section{Total antioxidant capacity (T-AOC) activity assay}

T-AOC activity was determined using a T-AOC assay kit (Nanjing Jiancheng Bioengineering Institute, Jiangsu, China) according to the manufacturer's instructions. The T-AOC activity was measured at $520 \mathrm{~nm}$ using a spectrophotometer (Shanghai Spectrophotometer Co., Ltd., Shanghai, China). Finally, T-AOC activity of each semen sample $\left(10^{7}\right.$ spermatozoa $\left./ \mathrm{ml}\right)$ was converted into units per $\mathrm{ml}(\mathrm{UI} / \mathrm{ml})$ of total protein in spermatozoa.

\section{Malondialdehyde (MDA) content assay}

Malondialdehyde content was measured with 2-thiobarbituric acid by monitoring the absorbance change at $532 \mathrm{~nm}$ with the spectrophotometer (Shanghai Spectrophotometer Co., Ltd., Shanghai, China) according to the instructions of the MDA kit (Nanjing Jiancheng Bioengineering Institute, Jiangsu, China). MDA content of spermatozoa samples $\left(10^{7}\right.$ spermatozoa $\left./ \mathrm{ml}\right)$ were expressed as $\mathrm{nmol} / \mathrm{l}$.

\section{Sperm capacitation and chlortetracycline (CTC) staining}

\section{Sperm capacitation}

Semen samples were centrifuged $(800 \times g, 10 \mathrm{~min}$ at $17^{\circ} \mathrm{C}$ ) and the pellets were diluted to a concentration of $1.5 \times 10^{7}$ cells per $\mathrm{mL}$ in Tyrode's medium $(100 \mathrm{mM}$ $\mathrm{NaCl}, 21.7 \mathrm{mM}$ lactate, $20 \mathrm{mM}$ Hepes, $15 \mathrm{mM} \mathrm{NaHCO}_{3}$, $5 \mathrm{mM}$ glucose, $3.1 \mathrm{mM} \mathrm{KCl}, 2.0 \mathrm{mM} \mathrm{CaCl}, 1.0 \mathrm{mM}$ pyruvate, $0.4 \mathrm{mM} \mathrm{MgSO}$, $0.3 \mathrm{mM} \mathrm{NaH}_{2} \mathrm{PO}_{4}$, and $50 \mathrm{mg} /$ $\mathrm{ml}$ kanamycin, $\mathrm{pH}$ 7.4) with $3 \mathrm{mg} / \mathrm{L}$ bovine serum albumin (BSA). Samples were then was incubated for $3 \mathrm{~h}$ in a humidified incubator in an atmosphere of $5 \% \mathrm{CO}_{2}$ at 37 ${ }^{\circ} \mathrm{C}$ to induce in vitro capacitation $[10,50]$.

\section{Sperm CTC staining}

The CTC staining procedure was essentially carried out as described [43]. In brief, CTC (750 pM) was prepared in a buffer of $(\mathrm{mM}) 20$ Tris, $130 \mathrm{NaCl}$ and $5 \mathrm{DL}$ cysteine (final $\mathrm{pH}$ 7.8). Five $\mu \mathrm{L}$ of sperm suspension was mixed with $5 \mu \mathrm{L} \mathrm{CTC}$ on a clean warm slide; after $30 \sec 5$ $\mu \mathrm{L}$ of $0.2 \%$ gluteraldehyde solubilized in $0.5 \mathrm{M}$ Tris buffer (final $\mathrm{pH}$ 7.4) was added. One drop of $0.22 \mathrm{M} 1$, 4-diazabicyclo $[2,2,2]$ octane dissolved in glycerol:phosphatebuffered saline $(9: 1)$ was added to retard the fading of CTC fluorescence. After adding a clean coverslip, 200 spermatozoa per slide were observed using a fluorescence microscope equipped with phase contrast and green epifluorescent optics (Nikon, Japan).

\section{Western blot analysis}

Exosomes pellets were lysed using RIPA buffer containing $1 \%$ protease inhibitor cocktail (Cat NO. BML-KI103-0001, Enzo Life Sciences, USA). Lysates were centrifuged at $14,000 \times g$ for $20 \mathrm{~min}$. Supernatant fractions were electrophoresed on a $10 \%$ SDS-PAGE gel and transferred to $0.45-\mu \mathrm{m}$ PVDF membranes (Millipore, USA). Then the membranes were blocked using 5\% $(\mathrm{w} / \mathrm{v})$ skim milk for $1 \mathrm{~h}$ and washed three times in TBST. The membranes were incubated overnight at $4{ }^{\circ} \mathrm{C}$ in a primary antibody solution containing anti-CD9 from goat polyclonal antibody (Cat.No. Sc7639; Santa Cruz), antiCD63 (Cat. NO. sc-15363, Santa Cruz) and anti-AWN (see Table S1 and Figure S1) at a dilution of 1:1000. After washing with TBST, the membrane was incubated in the goat anti-rabbit IgG (or the monkey anti-goat IgG for CD9) coupled to horseradish peroxidase $(1: 10,000)$ and for $1 \mathrm{~h}$ at room temperature. The membrane was then incubated 5 min with ECL and chemiluminescence was detected using the BIO-RAD ChemiDoc XRS Imaging system.

\section{Indirect immunofluorescence}

Sperm samples were washed three times with PBS at $800 \times g$ for $5 \mathrm{~min}$ at room temperature. Sperm suspension $\left(1 \times 10^{7}\right.$ sperm $\left./ \mathrm{ml}\right)$ was fixed in $4 \%(\mathrm{w} / \mathrm{v})$ paraformaldehyde (PBS/PFA) for $30 \mathrm{~min}$. After washing three times with PBS, the sperm were resuspended in PBS and placed onto poly-L-lysine-coated coverslips $(5-\mu \mathrm{L}$ sperm suspension/spot), and completely dried for $15 \mathrm{~min}$ at room temperature. The sperm spots were then washed three times with PBS before immunofluorescence staining. Briefly, sperm spots were incubated for $30 \mathrm{~min}$ at $37^{\circ} \mathrm{C}$ in PBS supplemented with $2 \%$ skim milk (vlv). Spermatozoa were incubated overnight at $4^{\circ} \mathrm{C}$ with the primary antibody to AWN and PSP-1 (see Table S1 and Figure S1). Identical samples incubated in parallel in PBS supplemented with rabbit preimmune serum were used as negative controls. After overnight incubation in primary antibody, the slides were washed extensively with PBS and incubated for $30 \mathrm{~min}$ at $37^{\circ} \mathrm{C}$ with the secondary antibody (FITCconjugated polyclonal antibody to rabbit $\mathrm{IgG}$ ). The slides were then incubated with DAPI for 2 min. Finally, the slides were washed three times with PBS, and examined using a fluorescent microscope.

\section{Scanning electron microscopy}

Two $\mathrm{mL}$ of sperm sample were fixed in $2.5 \%$ glutaraldehyde in $0.01 \mathrm{M}$ phosphate buffered saline buffer (PBS) for $1 \mathrm{~h}$ at $4^{\circ} \mathrm{C}$. After the fixation, the samples were centrifuged at $800 \times g$ for $2 \mathrm{~min}$ and then washed three 
times with PBS. Following mounting on silicon slice and air drying, sperm samples were post-fixed in $0.2 \%$ aqueous osmium tetroxide for $30 \mathrm{~min}$ and then dehydrated in increasing concentrations of acetone $(35,50,75,95$ and $100 \%$ ) was followed by critical point drying. One $\mathrm{mL}$ of exosome solution was directly dropped onto a silicon slice and air dried. Finally, the specimens were sputter-coated with gold palladium and viewed under a scanning electron microscope (Leo 1455 VP, Cambridge, UK).

\section{Statistical analyses}

All analyses were performed using Statistical Product and Service Solutions (SPSS 11.5 for windows; SPSS, Chicago, IL, USA). All results were expressed as mean $\mathrm{SD}$. The mean values of the percentages of motile sperm, dead sperm, plasma membrane-intact sperm and T-AOC level and MDA content were compared using Duncan's multiple range tests by ANOVA procedure, when the $\mathrm{F}$ value was significant $(p<0.05)$. The correlation among the above-mentioned methods was evaluated by linear regression analysis.

\section{ACKNOWLEDGMENTS}

We are indebted to Prof. Wenxian Zeng and Prof. Gongshe Yang at Northwest A \& F University for comments and suggestion of the study. We also thanked that the editors of the Aisiai Editing Services edited the manuscript in English language, grammar, and overall style.

\section{CONFLICTS OF INTEREST}

All authors read and approved the final manuscript. The authors declare no competing financial interests.

\section{GRANT SUPPORT}

This study was supported in part by the National Natural Science Foundation of China (Grant NO.C17010431172205 and NO.C170104-31572401) and the National Swine Industry Technology System Foundation (CARS36).

\section{REFERENCES}

1. Koch S, Acebron SP, Herbst J, Hatiboglu G, Niehrs C. Posttranscriptional Wnt Signaling Governs Epididymal Sperm Maturation. Cell. 2015; 163:1225-1236.

2. Poliakov A, Spilman M, Dokland T, Amling CL, Mobley JA. Structural heterogeneity and protein composition of exosome-like vesicles (prostasomes) in human semen. The Prostate. 2009; 69:159-167.
3. Suzuki K, Asano A, Eriksson B, Niwa K, Nagai T, Rodriguez-Martinez H. Capacitation status and in vitro fertility of boar spermatozoa: effects of seminal plasma, cumulus-oocyte-complexes-conditioned medium and hyaluronan. International journal of andrology. 2002; 25:84-93.

4. Abney TO, Williams WL. Inhibition of sperm capacitation by intrauterine deposition of seminal plasma decapacitation factor. Biology of reproduction. 1970; 2:14-17.

5. Mendoza N, Casao A, Perez-Pe R, Cebrian-Perez JA, Muino-Blanco T. New insights into the mechanisms of ram sperm protection by seminal plasma proteins. Biology of reproduction. 2013; 88:149.

6. Huo LJ, Yue KZ, Yang ZM. Characterization of viability, mitochondrial activity, acrosomal integrity and capacitation status in boar sperm during in vitro storage at different ambient temperatures. Reproduction, fertility, and development. 2002; 14:509-514.

7. Kovak MR, Saraswati S, Schoen DJ, Diekman AB. Investigation of galectin-3 function in the reproductive tract by identification of binding ligands in human seminal plasma. Am J Reprod Immunol. 2014; 72:403-412.

8. Sullivan R, Saez F, Girouard J, Frenette G. Role of exosomes in sperm maturation during the transit along the male reproductive tract. Blood cells, molecules \& diseases. 2005; 35:1-10.

9. Gatti JL, Metayer S, Belghazi M, Dacheux F, Dacheux JL. Identification, proteomic profiling, and origin of ram epididymal fluid exosome-like vesicles. Biology of reproduction. 2005; 72:1452-1465.

10. Piehl LL, Fischman ML, Hellman U, Cisale H, Miranda PV. Boar seminal plasma exosomes: effect on sperm function and protein identification by sequencing. Theriogenology. 2013; 79:1071-1082.

11. Park KH, Kim BJ, Kang J, Nam TS, Lim JM, Kim HT, Park JK, Kim YG, Chae SW, Kim UH. Ca2+ signaling tools acquired from prostasomes are required for progesteroneinduced sperm motility. Sci Signal. 2011; 4: ra31.

12. Aalberts M, Stout TA, Stoorvogel W. Prostasomes: extracellular vesicles from the prostate. Reproduction. 2014; 147:1-14.

13. Berruti G, Paiardi C. Acrosome biogenesis: Revisiting old questions to yield new insights. Spermatogenesis. 2011; 1:95-98.

14. Zhang HG, Cao P, Teng Y, Hu X, Wang Q, Yeri AS, Zhuang X, Samykutty A, Mu J, Deng ZB et al. Isolation, identification, and characterization of novel nanovesicles. Oncotarget. 2016; 7:41364-41362. doi: 10.18632/ oncotarget.9325.

15. Royo F, Zuniga-Garcia P, Sanchez-Mosquera P, Egia A, Perez A, Loizaga A, Arceo R, Lacasa I, Rabade A, Arrieta E et al. Different EV enrichment methods suitable for clinical settings yield different subpopulations of urinary extracellular vesicles from human samples. Journal of 
extracellular vesicles. 2016; 5: 1-11.

16. Sanz L, Calvete JJ, Mann K, Schafer W, Schmid ER, Amselgruber W, Sinowatz F, Ehrhard M, Topfer-Petersen E. The complete primary structure of the spermadhesin AWN, a zona pellucida-binding protein isolated from boar spermatozoa. FEBS letters. 1992; 300:213-218.

17. Petrunkina AM, Harrison RA, Topfer-Petersen E. Only low levels of spermadhesin AWN are detectable on the surface of live ejaculated boar spermatozoa. Reproduction, fertility, and development. 2000; 12:361-371.

18. Caballero I, Vazquez JM, Garcia EM, Roca J, Martinez EA, Calvete JJ, Sanz L, Ekwall H, Rodriguez-Martinez H. Immunolocalization and possible functional role of PSP-I/ PSP-II heterodimer in highly extended boar spermatozoa. Journal of andrology. 2006; 27:766-773.

19. Saez F, Sullivan R. Prostasomes, post-testicular sperm maturation and fertility. Front Biosci (Landmark Ed.) 2016; 21:1464-1473.

20. Higgins SJ, Burchell JM, Mainwaring WI. Androgendependent synthesis of basic secretory proteins by the rat seminal vesicle. The Biochemical journal. 1976; 158:271282.

21. Davis BK. Decapacitation and recapacitation of rabbit spermatozoa treated with membrane vesicles from seminal plasma. J Reprod Fertil. 1974; 41:241-244.

22. Breitbart $\mathrm{H}$, Rubinstein S. Characterization of $\mathrm{Mg} 2+-$ and $\mathrm{Ca} 2+-\mathrm{ATPase}$ activity in membrane vesicles from ejaculated ram seminal plasma. Arch Androl. 1982; 9:147157.

23. Killian GJ, Chapman DA, Rogowski LA: Fertilityassociated proteins in Holstein bull seminal plasma. Biology of reproduction. 1993; 49:1202-1207.

24. Sostaric E, Aalberts M, Gadella BM, Stout TA: The roles of the epididymis and prostasomes in the attainment of fertilizing capacity by stallion sperm. Anim Reprod Sci. 2008; 107:237-248.

25. Simons M, Raposo G: Exosomes-vesicular carriers for intercellular communication. Curr Opin Cell Biol. 2009, 21:575-581.

26. Thery C, Zitvogel L, Amigorena S: Exosomes: composition, biogenesis and function. Nature reviews Immunology. 2002, 2:569-579.

27. Ronquist G, Frithz GG: Prostasomes in human semen contain ADP and GDP. Acta Europaea fertilitatis. 1986; 17:273-276.

28. Pons-Rejraji H, Artonne C, Sion B, Brugnon F, Canis M, Janny L, Grizard G. Prostasomes: inhibitors of capacitation and modulators of cellular signalling in human sperm. International journal of andrology. 2011; 34:568-580.

29. Hernandez M, Roca J, Calvete JJ, Sanz L, Muino-Blanco T, Cebrian-Perez JA, Vazquez JM, Martinez EA. Cryosurvival and in vitro fertilizing capacity postthaw is improved when boar spermatozoa are frozen in the presence of seminal plasma from good freezer boars. Journal of andrology.
2007; 28:689-697.

30. Kareskoski AM, del Alamo MM, Guvenc K, Reilas T, Calvete JJ, Rodriguez-Martinez H, Andersson M, Katila T. Protein composition of seminal plasma in fractionated stallion ejaculates. Reprod Domest Anim. 2011; 46:e79-84.

31. Burden HP, Holmes CH, Persad R, Whittington K. Prostasomes - their effects on human male reproduction and fertility. Hum Reprod Update. 2006; 12:283-292.

32. Neild DM, Gadella BM, Chaves MG, Miragaya MH, Colenbrander B, Aguero A. Membrane changes during different stages of a freeze-thaw protocol for equine semen cryopreservation. Theriogenology. 2003; 59:1693-1705.

33. Johnson LA, Weitze KF, Fiser P, Maxwell WM. Storage of boar semen. Anim Reprod Sci. 2000; 62:143-172.

34. Agarwal A, Makker K, Sharma R. Clinical relevance of oxidative stress in male factor infertility: an update. Am J Reprod Immunol. 2008; 59:2-11.

35. Sariozkan S, Turk G, Canturk F, Yay A, Eken A, Akcay A. The effect of bovine serum albumin and fetal calf serum on sperm quality, DNA fragmentation and lipid peroxidation of the liquid stored rabbit semen. Cryobiology. 2013; 67:1-6.

36. Aitken RJ. Sperm function tests and fertility. International journal of andrology. 2006; 29:69-75.

37. Zhang XG, Yan GJ, Hong JY, Su ZZ, Yang GS, Li QW, Hu JH. Effects of bovine serum albumin on boar sperm quality during liquid storage at 17 degrees C. Reprod Domest. Anim 2015; 50:263-269.

38. Yamazaki D, Funato Y, Miyata H, Ikawa M, Miki H. Complementary role of CNNM2 in sperm motility and $\mathrm{Ca}(2+)$ influx during capacitation. Biochemical and biophysical research communications. 2016; 474:441-446.

39. Cisneros-Mejorado A, Hernandez-Soberanis L, IslasCarbajal MC, Sanchez D. Capacitation and $\mathrm{Ca}(2+)$ influx in spermatozoa: role of $\mathrm{CNG}$ channels and protein kinase G. Andrology. 2014; 2:145-154.

40. Kawakami E, Nishizono Y, Miyata C, Hirano T, Hori T, Tsutsui T. Effects of hyaluronic acid on $\mathrm{Ca}(2+)$ influx, lactate dehydrogenase activity, and cyclic AMP synthesis in canine ejaculated sperm during In Vitro capacitation. J Vet Med Sci. 2006; 68:119-123.

41. Kaul G, Sharma GS, Singh B, Gandhi KK. Capacitation and acrosome reaction in buffalo bull spermatozoa assessed by chlortetracycline and Pisum sativum agglutinin fluorescence assay. Theriogenology. 2001; 55:1457-1468.

42. DasGupta S, Mills CL, Fraser LR. Ca(2+)-related changes in the capacitation state of human spermatozoa assessed by a chlortetracycline fluorescence assay. J Reprod Fertil. 1993; 99:135-143.

43. Ward CR, Storey BT: Determination of the time course of capacitation in mouse spermatozoa using a chlortetracycline fluorescence assay. Dev Biol. 1984; 104:287-296.

44. Fabiani R, Johansson L, Lundkvist O, Ronquist G. Prolongation and improvement of prostasome promotive effect on sperm forward motility. European journal of 
obstetrics, gynecology, and reproductive biology. 1995; 58:191-198.

45. Davis BK. Timing of fertilization in mammals: sperm cholesterol/phospholipid ratio as a determinant of the capacitation interval. Proceedings of the National Academy of Sciences of the United States of America. 1981; 78:75607564.

46. Cohen R, Mukai C, Travis AJ. Lipid Regulation of Acrosome Exocytosis. Advances in anatomy, embryology, and cell biology. 2016; 220:107-127.

47. Hu JH, Li QW, Jiang ZL, Yang H, Zhang SS, Zhao HW. The cryoprotective effect of trehalose supplementation on boar spermatozoa quality. Reprod Domest Anim. 2009; 44:571-575
48. Estienne MJ, Harper AF, Day JL. Characteristics of sperm motility in boar semen diluted in different extenders and stored for seven days at 18 degrees C. Reprod Biol. 2007; 7:221-231.

49. Nagy S, Jansen J, Topper EK, Gadella BM. A triple-stain flow cytometric method to assess plasma- and acrosomemembrane integrity of cryopreserved bovine sperm immediately after thawing in presence of egg-yolk particles. Biology of reproduction. 2003; 68:1828-1835.

50. Flesch FM, Colenbrander B, van Golde LM, Gadella BM. Capacitation induces tyrosine phosphorylation of proteins in the boar sperm plasma membrane. Biochemical and biophysical research communications. 1999; 262:787-792. 